\title{
Editorial
}

\section{A new layout and update for Mineralogical Magazine in 2019}

\author{
Stuart J. Mills, Roger H. Mitchell and Helen C. Kerbey \\ Mineralogical Magazine, The Mineralogical Society of Great Britain and Ireland, 12 Baylis Mews, Amyand Park Road, Twickenham TW1 3HQ, UK
}

\section{Introduction}

The Mineralogical Society of Great Britain and Ireland has been proud to produce Mineralogical Magazine since the first issue was published in August 1876. The contents then included an article on a new form of blowpipe lamp, especially suitable for travelling mineralogists (Le Neve Foster, 1876) and thoughts on some basalt dredged up from the Atlantic by a telegraph cable-laying boat (Hall, 1876). Now with issue 544 we are discussing transmission electron microscopy (Nzogang et al., 2019) and testing the rocks on Mars (Larre et al., 2019).

In 2017, we successfully transferred the journal from print and on-line, to an on-line only publication, although printed versions are available on demand. In 2018 we commenced a publishing partnership with Cambridge University Press and as a consequence have increased the audience size for our periodical. The collaboration with CUP has also resulted in the release of our journal archive back to the first issue of the journal on Cambridge Core at https://www.cambridge.org/core/journals/ mineralogical-magazine.

The submission process for authors has been significantly improved by the use of the manuscript-tracking system Editorial Manager, at https://www.editorialmanager.com/minmag/default.aspx.

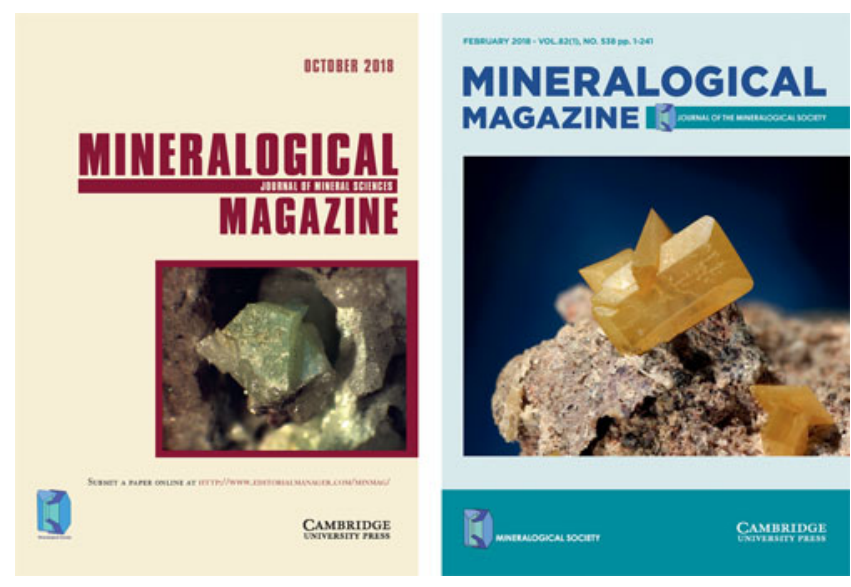

Fig. 1. The old and new covers for 2018 and 2019, respectively.

Author for correspondence: Stuart Mills, Email: SMills@museum.vic.gov.au Cite this article: Mills S.J., Mitchell R.H. and Kerbey H.C. (2019) A new layout and update for Mineralogical Magazine in 2019. Mineralogical Magazine, 83, 1-2. https:// doi.org/10.1180/mgm.2019.5

(C) Mineralogical Society of Great Britain and Ireland 2019
For 2019, we are pleased to announce some changes to the journal and to update some of our policies with respect to the data needed for submission of a manuscript to the journal.

\section{Changes in appearance}

- From 2019 we will have a new cover design (Fig. 1), and are changing size to A4 in order to better accommodate data tables;

- The journal will use British English as standard;

- References have a new format with no commas after surnames e.g. Mills S.J., Mitchell R.H. and Kerbey H.C. (2018);

- All crystal structures submitted must include a CIF and checkCIF report (https://checkcif.iucr.org/);

- Crystallographic tables should be submitted in a standardised format (see Tables 1-4);

\section{Changes to policy}

- Mineralogical Magazine strongly advises that representative specimens used in research are registered, if possible, and are housed in a suitable repository such as a geoscience collection in a museum. Registration numbers must be cited in the publication.

Table 1. Data and experimental details for the crystal selected.*

\begin{tabular}{|c|c|}
\hline \multicolumn{2}{|l|}{ Crystal data } \\
\hline Ideal formula & $\mathrm{Cu}_{16} \mathrm{Fe}_{8} \mathrm{Hg}_{29} \mathrm{~S}_{2}$ \\
\hline Crystal dimensions $(\mathrm{mm})$ & $0.020 \times 0.030 \times 0.060$ \\
\hline Crystal system, space group & Triclinic, $P \overline{1}$ \\
\hline Temperature $(\mathrm{K})$ & $293(3)$ \\
\hline$a, b, c(\AA)$ & 9.677(4), 9.865(5), 11.992(5) \\
\hline$\alpha, \beta, \gamma\left({ }^{\circ}\right)$ & $77.85(4), 79.42(3), 76.30(4)$ \\
\hline$V\left(\AA^{3}\right)$ & $1076.5(8)$ \\
\hline$z$ & 1 \\
\hline Calculated density $\left(\mathrm{g} \mathrm{cm}^{-3}\right)$ & 5.035 \\
\hline$\mu\left(\mathrm{mm}^{-1}\right)$ & 14.637 \\
\hline \multicolumn{2}{|l|}{ Data collection } \\
\hline Crystal description & Green partial hexagonal plate \\
\hline Instrument & Oxford Diffraction Xcalibur PX Ultra \\
\hline Radiation type, wavelength $(\AA ̊)$ & CuK $\alpha, 1.54184$ \\
\hline Number of frames & 1905 \\
\hline$\theta$ range $\left({ }^{\circ}\right)$ & 3.093 to 23.241 \\
\hline Absorption correction & Multi-scan (ABSPACK; Oxford Diffraction, 2006) \\
\hline$T_{\min }, T_{\max }$ & $0.4323,0.7464$ \\
\hline $\begin{array}{l}\text { No. of measured, independent and } \\
\text { observed }[I>2 \sigma(I)] \text { reflections }\end{array}$ & $4092,832,786$ \\
\hline$R_{\text {int }}$ & 0.0750 \\
\hline Data completeness to $23.241^{\circ} \theta(\%)$ & 99.4 \\
\hline Indices range of $h, k, l$ & $-10 \leq h \leq 11,-11 \leq k \leq 11,-12 \leq l \leq 13$ \\
\hline \multicolumn{2}{|l|}{ Refinement } \\
\hline Refinement & Full-matrix least squares on $F^{2}$ \\
\hline Number of reflections, parameters, restraints & $832,102,1$ \\
\hline$R_{1}[I>2 \sigma(I)], R_{1}$ (all) & $0.0296,0.0329$ \\
\hline $\mathrm{w} R_{2}[I>2 \sigma(I)], \mathrm{w} R_{2}$ (all) & $0.0608,0.0625$ \\
\hline GoF & 1.07 \\
\hline No. of refined parameters & 143 \\
\hline$\Delta \rho_{\max }, \Delta \rho_{\min }\left(e^{-} \AA^{-3}\right)$ & $1.34,-1.22$ \\
\hline
\end{tabular}

*Various miscellaneous entries, placed on the same line where possible, and using the same notation as above. 
Table 2. Atom coordinates and displacement parameters. *

\begin{tabular}{|c|c|c|c|c|c|c|c|c|c|c|}
\hline & $x / a$ & $y / b$ & $z / c$ & $U_{\text {eq }}$ & $U^{11}$ & $U^{13}$ & $U^{23}$ & $U^{22}$ & $U^{33}$ & $U^{12}$ \\
\hline $\mathrm{Na} 1$ & $0.7160(12)$ & $-0.0931(9)$ & $0.3631(5)$ & $0.035(3)$ & $0.036(5)$ & $0.0002(3)$ & $0.0005(3)$ & $0.022(5)$ & $0.047(6)$ & $0.007(3)$ \\
\hline $\mathrm{Na} 2$ & $1 / 4$ & $0.8584(10)$ & $1 / 4$ & $0.027(3)$ & $0.017(4)$ & $0.0002(3)$ & $0.0005(3)$ & $0.031(5)$ & $0.033(5)$ & 0 \\
\hline$U$ & $0.74439(4)$ & $0.40606(4)$ & $0.36948(2)$ & $0.01707(11)$ & $-0.01353(16)$ & $0.0004(3)$ & $0.0005(3)$ & $0.02393(18)$ & $0.01374(16)$ & $0.00003(12)$ \\
\hline $\mathrm{S}$ & $0.2468(3)$ & $0.5059(3)$ & $0.41111(11)$ & $0.0210(4)$ & $-0.0131(9)$ & $0.0002(3)$ & $0.0005(3)$ & $0.0384(13)$ & $0.0115(8)$ & $0.0003(8)$ \\
\hline
\end{tabular}

*Ideally combined in a single table, will be aligned on the decimal point.

Table 3. Powder X-ray data.*

\begin{tabular}{rrllr}
\hline$I_{\text {obs }}$ & $I_{\text {calc }}$ & $d_{\text {obs }}$ & \multicolumn{1}{c}{$d_{\text {calc }}$} & $h k l$ \\
\hline 41 & 24 & 5.80 & 5.818 & 002 \\
14 & 14 & 5.65 & 5.669 & 021 \\
35 & 21 & 5.31 & 5.325 & 120 \\
7 & 2 & 4.65 & 4.655 & 200 \\
27 & 14 & 4.42 & 4.415 & 202 \\
37 & 27 & 3.916 & 3.920 & 221 \\
4 & 3 & 3.584 & 3.604 & 122 \\
$\mathbf{9 8}$ & $\mathbf{5 7}, \mathbf{2 7}$ & $\mathbf{3 . 3 3 9}$ & $\mathbf{3 . 3 4 3}, \mathbf{3 . 3 3 0}$ & $\mathbf{2 2 1}$ \\
65 & 58 & 3.155 & 3.161 & 202 \\
$\mathbf{1 0 0}$ & $\mathbf{1 0 0}$ & $\mathbf{3 . 0 4 3}$ & $\mathbf{3 . 0 4 6}$ & $\mathbf{1} \mathbf{4 1}$ \\
72 & 68 & 2.940 & 2.942 & $\overline{\mathbf{2}} 04$ \\
34 & 22 & 2.879 & 2.881 & $320, \overline{\mathbf{1}} 24$ \\
51 & 21,22 & 2.787 & $2.800,2.784$ &
\end{tabular}

* $d_{\mathrm{obs}}$ and $d_{\text {calc }}$ data adjacent to each other, multiple peaks on the same line and the strongest lines $(\max 8)$ in bold

- Type specimens must have registration numbers. Where a new crystal structure is reported or a species is redefined, registration numbers must be given.

- It is advisable to identify all samples (images and data) with specimen numbers.

We hope that you enjoy the new format and we look forward to publishing your best research in Mineralogical Magazine!
Table 4. Selected interatomic distances $(\AA ̊) .^{*}$

\begin{tabular}{llll}
\hline Al1-O1 & $1.908(4) \times 2$ & $\mathrm{Pb3}-\mathrm{OH} 2$ & $2.23(1)$ \\
Al1-O2 & $1.913(3) \times 2$ & $\mathrm{Pb3}-\mathrm{O} 3$ & $2.688(2) \times 6$ \\
Al1-O3 & $1.999(3) \times 2$ & $\mathrm{Pb3}-\mathrm{O} 1$ & $3.229(8) \times 3$ \\
$<\mathrm{Al}-\mathrm{O}>$ & 1.940 & $<\mathrm{Pb3}-\mathrm{O}>$ & $2.500(2)$ \\
& & & \\
$\mathrm{Al} 2-\mathrm{O} 1$ & $1.950(4) \times 6$ & $\mathrm{Na}-\mathrm{OW}(2)$ & $2.10(2)$ \\
$<\mathrm{Al2}-\mathrm{O}>$ & 1.950 & $\mathrm{Na}-\mathrm{O}(4)$ & $2.484(8) \times 2$ \\
& & $\mathrm{Na}-\mathrm{O}(4)$ & $2.779(11) \times 2$ \\
& & $<\mathrm{Na}-\mathrm{O}>$ & $2.488(3)$ \\
\hline
\end{tabular}

${ }^{\star}$ Full atom details, empty lines between each group.

\section{References}

Hall M. (1876) I. Note upon a portion of Basalt from Mid-Atlantic. Mineralogical Magazine and Journal of the Mineralogical Society, 1(1), 1-3.

Larre C., Morizet Y., Guillot-Deudon C., Baron F. and Mangold N. (2019) Quantitative Raman calibration of sulfate-bearing polymineralic mixtures: a $S$ quantification in sedimentary rocks on Mars. Mineralogical Magazine, https:// doi.org/10.1180/mgm.2018.147

Le Neve Foster C. (1876) VII. Description of a new form of blowpipe lamp, especially suitable for travellers. Mineralogical Magazine and Journal of the Mineralogical Society, 1(1), 20.

Nzogang B.C., Mussi A. and Cordier P. (2019) Application of Scanning Electron Diffraction in the Transmission Electron Microscope for the characterization of dislocations in minerals. Mineralogical Magazine, https://doi.org/10.1180/mgm.2018.144 